\title{
MODELING OF THE NEUTRAL AXES OF A CIRCULAR PIEZOELECTRIC MICROMACHINED TRANSDUCER IN TRANSMIT AND RECEIVE MODE
}

\author{
Firas Sammoura, ${ }^{1,2}{ }^{*}$ and Sang-Gook Kim²
}

${ }^{1}$ Microsystems Division, Masdar Institute of Science and Technology, Abu Dhabi, UAE

${ }^{2}$ Department of mechanical engineering, Massachusetts Institute of Technology, Cambridge, MA 02139, USA

\begin{abstract}
A theoretical model that predicts the neutral axes locations of a circular Piezoelectric Micromachined Ultrasonic Transducer (PMUT) in both transmit and receive mode has been successfully developed for the first time. The constitutive vibration equations of plates in polar coordinates were used to analyze the bending of the circular plate forming the PMUT device. A general expression for the neutral axis position, a planar section with zero longitudinal stresses and strains, was derived as a function of the externally applied bending moment, the radial force, and the electric field between the two metal electrodes. Whereas the piezoelectric layer of the PMUT is subjected to an electric field during the transmit mode, the two metal electrodes are grounded during the receive mode. As such, distinct locations of the neutral axes in transmit and receive mode are predicted and verified using Finite Element Modeling (FEM). This unprecedented demonstration opens up new opportunities for accurate modeling of PMUTs and precise extraction of performance parameters.
\end{abstract}

\section{INTRODUCTION}

Ultrasonic sensors have been used as medical diagnostic tools to image anatomy for the past 50 years [1]. Thickness mode piezoelectric resonators operating in a high frequency range (2-15 $\mathrm{MHz}$ ) convert electrical pulses to sound waves and vice versa [2]. In order to design a transducer with high sensitivity and compact impulse response, it is necessary to engineer a piezoelectric composite with enhanced electromechanical coupling and acoustic impedance that closely matches with the tissue [3]. However, the dice-and-fill technique currently used to combine piezoelectric ceramics and passive polymers to form a piezocomposite ultrasonic array is tedious.

With the advancement of microelectromechanical systems (MEMS), Capacitive Micromachined Ultrasonic Transducers (CMUTs) have been introduced as a substitute to the traditional bulk piezoelectric transducers, with enhanced bandwidth and sensitivity [4]. However, CMUTs suffer from limited output acoustic power, high bias voltage, and non-linear plate deflection with applied electromotive force [5].

PMUTs have been lately introduced as an alternative to CMUTs [6]. Unlike CMUT, a PMUT is not subject to the geometric constraints of electrostatic transduction, enabling higher acoustic pressure output and energy efficiency. A typical PMUT is a suspended plate clamped at its edges resulting in reduced device acoustic impedance. The unimorph plate is composed of a structural layer and a piezoelectric material sandwiched between two electrodes. The membrane vibrates with the application of AC voltage due to the piezoelectric phenomenon. Specifically, Shelton et al. have demonstrated an air-coupled PMUT using Aluminum Nitride (AIN) as a piezoelectric material [7], and Muralt et al. characterized a PMUT fabricated with a $2 \mu \mathrm{m}$ thick PZT film [8]. There have also been some efforts aimed at theoretically modeling PMUTs. Bernstein et al. reported simple analytical formulae that predict the resonant frequency of a circular membrane suspended in air or loaded at one-side under simply supported and clamped boundary conditions [9]. Perçin et al. outlined the equations governing the operation of a circular PMUT and attempted to derive a circuit model for piezoelectrically actuated micromachined ultrasound transducers. However, the previous modeling work neither touched on the unimorph nature of a PMUT, nor on the effect of the neutral axis location on the circuit parameters [10].

Determining the neutral axis location is a precursor for evaluating the static and dynamic response of a unimorph PMUT. Recently, DeVoe et al. described a model that predicts the static behavior of a piezoelectric cantilever actuator with arbitrary configuration of piezoelectric and structural layers [11]. Weinberg et al. derived solution to the force, displacements, and charges in piezoelectric beams based on determining the neutral axis location [12]. In contrast to the previous works, this is the first paper that investigates the exact location of the neutral axis of a circular unimorph PMUT and distinguishes between the operation in transmit and receive modes.

\section{(a) Before Actuation}
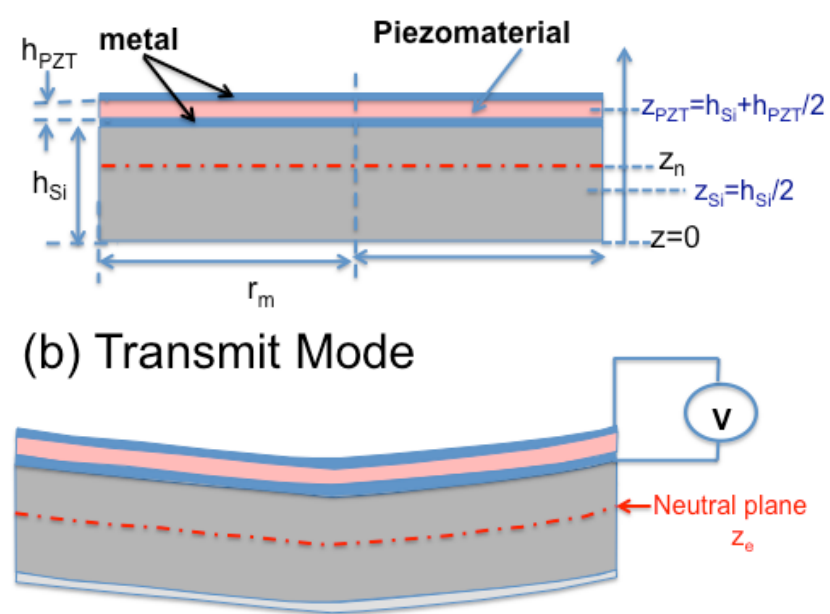

\section{(c) Receive Mode}

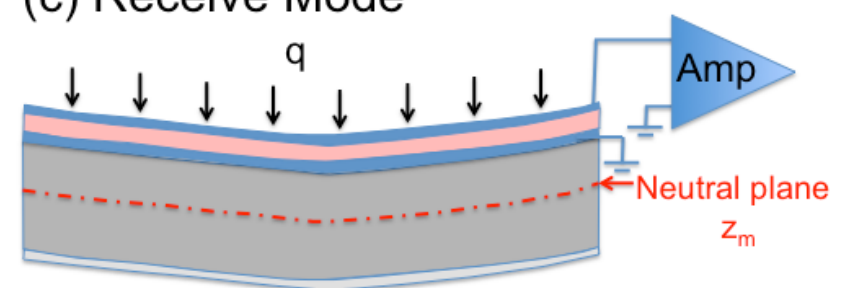

Figure 1: A schematic diagram of a Piezoelectric Micromachined Ultrasonic Transducer (PMUT) composed of a structural layer and a piezoelectric layer sandwiched between two metal electrodes: (a) before actuation, (b) in transmit mode with excitation potential $V$, and (c) in receive mode with an applied pressure of $q$.

\section{THEORY}

Overview of the Unimorph PMUT

Figure 1(a) shows a schematic diagram of the circular 
unimorph PMUT under consideration. The PMUT is composed of a structural layer of thickness $h_{S i}$ and a piezoelectric layer of thickness $h_{P Z T}$. The piezoelectric layer is sandwiched between two metal electrodes of negligible thickness. The plate radius is designated as $r_{m}$ and the neutral axis is located at a position $z_{n}$ from a reference $z=0$, set at the bottom of the plate. In the transmit mode represented in Figure 1(b), the piezoelectric layer is excited with an electric potential $V$. The strain mismatch between the structural and the piezoelectric layers causes an out-of-plane plate vibration and the generation of an ultrasonic wave. In the receive mode, an echo pressure wave impinges onto the plate as shown in Figure 1(c). The plate deflection leads to charge separation in the piezoelectric layer, which generates a current in the charge amplifier. The neutral axes' locations in transmit and receive modes are denoted as $z_{e}$ and $z_{m}$, respectively.

\section{Working Equations}

Classic plate theory is used to model the circular unimorph PMUT. The two-layer unimorph plate structure shown in Figure 1 is generalized into an n-layer multimorph, each layer of which can be either structural or piezoelectric. As the plate lateral dimensions are much larger than its thickness, in-plane stress conditions dominate. Both the structural and the piezoelectric layers are assumed to be transversely isotropic. The interfaces between the respective layers are considered non-slip surfaces. The strains $\varepsilon_{r r}$ and $\varepsilon_{\theta \theta}$ in the r-and $\theta$-directions, respectively, at a distance $z$ from the neutral axis position are thus continuous and can be measured as follows [13]:

$$
\begin{gathered}
\varepsilon_{r r}=\frac{z-z_{n}}{R_{r r}} \\
\varepsilon_{\theta \theta}=\frac{z-z_{n}}{R_{\theta \theta}}
\end{gathered}
$$

where $R_{r r}$ and $R_{\theta \theta}$ are the radii of curvature in the r- and $\theta$ directions, respectively. The principal strains and stresses in the $i^{\text {th }}$ layer are related to the materials properties and the applied electric field according to Hooke's law [13]:

$$
\left[\begin{array}{c}
\varepsilon_{r r, i} \\
\varepsilon_{\theta \theta, i}
\end{array}\right]=\frac{1}{Y_{0, i}}\left[\begin{array}{cc}
1 & -v_{i} \\
-v_{i} & 1
\end{array}\right]\left[\begin{array}{c}
T_{r r, i} \\
T_{\theta \theta, i}
\end{array}\right]+\left[\begin{array}{l}
d_{31, i} \\
d_{31, i}
\end{array}\right] E_{z, i}
$$

where $T_{r r, i}$ and $T_{\theta e, i}$ are the in-plane stresses in the $\mathrm{r}$ - and $\theta$ directions, respectively, $Y_{0, i}$ is the Young's modulus, $v_{i}$ is the Poisson's ratio, $d_{31, i}$ is transverse piezoelectric charge constant, and $E_{z, i}$ is the electric field applied along the $\mathrm{z}$-direction of the $\mathrm{i}^{\text {th }}$ layer. The stresses in the $r$ - and $\theta$-directions can be re-written in terms of the strains by inverting Eq. (3):

$$
\left[\begin{array}{c}
T_{r r, i} \\
T_{\theta \theta, i}
\end{array}\right]=Y_{0, i}^{\prime}\left(\left[\begin{array}{cc}
1 & v_{i} \\
v_{i} & 1
\end{array}\right]\left[\begin{array}{c}
\varepsilon_{r r, i} \\
\varepsilon_{\theta \theta, i}
\end{array}\right]-\left[\begin{array}{c}
d_{31, i}^{\prime} \\
d_{31, i}^{\prime}
\end{array}\right] E_{z, i}\right)
$$

where $Y_{0, i}{ }^{\prime}$ is the equivalent Young's modulus and $d_{31, i}$ is the equivalent transverse piezoelectric charge constant defined as follows:

$$
\begin{aligned}
& Y_{0, i}^{\prime}=\frac{Y_{0, i}}{1-v_{i}^{2}} \\
& d_{31, i}^{\prime}=\left(1+v_{i}\right) d_{31, i}
\end{aligned}
$$

The externally applied force per unit width in the radial direction, $N_{r}$, can be calculated by integrating the radial stresses over the PMUT thickness at any radial position as follows:

$$
N_{r r}=\sum_{i=1}^{n} \int_{h_{i-1}}^{h_{i}} T_{r r, i} d z
$$

By performing the integration as indicated by Eq. (7), the radial force can be expressed as follows:

$$
\begin{aligned}
N_{r r}=( & \left.\frac{1}{R_{r r}}\right)\left[\sum_{i=1}^{n} Y_{0, i}^{\prime} z_{i} h_{i}-z_{n} \sum_{i=1}^{n} Y_{0, i}^{\prime} h_{i}\right] \\
& +\left(\frac{1}{R_{\theta \theta}}\right)\left[\sum_{i=1}^{n} v_{i} Y_{0, i}^{\prime} z_{i} h_{i}-z_{n} \sum_{i=1}^{n} v_{i} Y_{0, i}^{\prime} h_{i}\right] \\
& -\sum_{i=1}^{n} Y_{0, i}^{\prime} d_{31, i}^{\prime} h_{i} E_{z, i}
\end{aligned}
$$

where $z_{i}$ is the distance measured from the z-reference to the middle of the $\mathrm{i}^{\text {th }}$ layer. The externally applied moment per unit width in the radial direction, $M_{r r}$, should be balanced by moments generated by the in-plane stresses around the z-reference. The moment is calculated using the following equation:

$$
M_{r r}=\sum_{i=1}^{n} \int_{h_{i-1}}^{h_{i}} T_{r r, i} z d z
$$

The following expression for $M_{r r}$ is obtained upon carrying out the integral of Eq. (9):

$$
\begin{aligned}
M_{r r}=( & \left.\frac{1}{R_{r r}}\right)\left[\sum_{i=1}^{n} Y_{0, i}^{\prime}\left(I_{i}+z_{i}^{2} h_{i}\right)-z_{n} \sum_{i=1}^{n} Y_{0, i}^{\prime} z_{i} h_{i}\right] \\
& +\left(\frac{1}{R_{\theta \theta}}\right)\left[\sum_{i=1}^{n} v_{i} Y_{0, i}^{\prime}\left(I_{i}+z_{i}^{2} h_{i}\right)-z_{n} \sum_{i=1}^{n} v_{i} Y_{0, i}^{\prime} z_{i} h_{i}\right] \\
& -\sum_{i=1}^{n} Y_{0, i}^{\prime} d_{31, i}^{\prime} z_{i} h_{i} E_{z, i}
\end{aligned}
$$

where $I_{i}$ is the second moment of area per unit width calculated around each layer's centerline as follows:

$$
I_{i}=\frac{h_{i}^{3}}{12}
$$

If we assume that the Poisson's ratio $v_{i}$ of all the layers, whether structural or piezoelectric, is the same and set its value equal to $v$, the externally applied radial force and moment per unit width can be rearranged using Eqs. (8) and (10) as follows: 


$$
\begin{aligned}
& N_{r r}=\left(\frac{1}{R_{e q}}\right)\left[\sum_{i=1}^{n} Y_{0, i}^{\prime} z_{i} h_{i}-z_{n} \sum_{i=1}^{n} Y_{0, i}^{\prime} h_{i}\right]-\sum_{i=1}^{n} Y_{0, i}^{\prime} d_{31, i}^{\prime} h_{i} E_{z, i} \\
& M_{r r}=\left(\frac{1}{R_{e q}}\right)\left[\sum_{i=1}^{n} Y_{0, i}^{\prime}\left(I_{i}+z_{i}^{2} h_{i}\right)-z_{n} \sum_{i=1}^{n} Y_{0, i}^{\prime} z_{i} h_{i}\right]-\sum_{i=1}^{n} Y_{0, i}^{\prime} d_{31, i}^{\prime} z_{i} h_{i} E_{z, i}
\end{aligned}
$$

where $R_{e q}$ is the equivalent radius of curvature due to the radii of curvature in the r-direction, $\theta$-direction, and the Poisson's ratio as follows:

$$
\frac{1}{R_{e q}}=\frac{1}{R_{r r}}+\frac{v}{R_{\theta \theta}}
$$

By eliminating the equivalent radius of curvature term in Eqs. (12) and (13), the neutral axis position can be finally expressed as a function of the externally applied radial moment and force per unit width as well as the electric fields applied across each piezoelectric layer as follows:

$$
z_{n}=\frac{\left[\sum_{i=1}^{n} Y_{0, i}^{\prime}\left(I_{i}+z_{i}^{2} h_{i}\right)\right]\left(N_{r r}+\sum_{i=1}^{n} Y_{0, i}^{\prime} d_{31, i}^{\prime} h_{i} E_{z, i}\right)-\left[\sum_{i=1}^{n} Y_{0, i}^{\prime} z_{i} h_{i}\right]\left(M_{r r}+\sum_{i=1}^{n} Y_{0, i}^{\prime} d_{31, i}^{\prime} z_{i} h_{i} E_{z, i}\right)}{\left[\sum_{i=1}^{n} Y_{0, i}^{\prime} z_{i} h_{i}\right]\left(N_{r r}+\sum_{i=1}^{n} Y_{0, i}^{\prime} d_{31, i}^{\prime} h_{i} E_{z, i}\right)-\left[\sum_{i=1}^{n} Y_{0, i}^{\prime} h_{i}\right]\left(M_{r r}+\sum_{i=1}^{n} Y_{0, i}^{\prime} d_{31, i}^{\prime} z_{i} h_{i} E_{z, i}\right)}
$$

\section{Neutral Axes Location}

Case I: Transmit mode

For the case of a PMUT in the transmit mode, there are no externally applied radial moments and forces. As such the neutral axis position $z_{e}$ reduces to the following:

$$
z_{e}=\frac{\left[\sum_{i=1}^{n} Y_{0, i}^{\prime}\left(I_{i}+z_{i}^{2} h_{i}\right)\right]\left(\sum_{i=1}^{n} Y_{0, i}^{\prime} d_{31, i}^{\prime} h_{i} E_{z, i}\right)-\left[\sum_{i=1}^{n} Y_{0, i}^{\prime} z_{i} h_{i}\right]\left(\sum_{i=1}^{n} Y_{0, i}^{\prime} d_{31, i}^{\prime} z_{i} h_{i} E_{z, i}\right)}{\left.\left[\sum_{i=1}^{n} Y_{0, i}^{\prime} z_{i} h_{i}\right]\right]\left(\sum_{i=1}^{n} Y_{0, i}^{\prime} d_{31, i}^{\prime} h_{i} E_{z, i}\right)-\left[\sum_{i=1}^{n} Y_{0, i}^{\prime} h_{i}\right]\left(\sum_{i=1}^{n} Y_{0, i}^{\prime} d_{31, i}^{\prime} z_{i} h_{i} E_{z, i}\right)}
$$

If there is only one piezoelectric layer in the PMUT structure, the neutral axis in the transmit mode is simplified to the following:

$$
z_{e 1}=\frac{\sum_{i=1}^{n} Y_{0, i}^{\prime}\left(I_{i}+z_{i}^{2} h_{i}\right)-z_{P} \sum_{i=1}^{n} Y_{0, i}^{\prime} z_{i} h_{i}}{\sum_{i=1}^{n} Y_{0, i}^{\prime} z_{i} h_{i}-z_{P} \sum_{i=1}^{n} Y_{0, i}^{\prime} h_{i}}
$$

where $z_{p}$ is the distance from the z-reference to the center of the piezoelectric layer.

\section{Case II: Receive mode}

When the PMUT operates in the receive mode, there are no electric fields across the respective piezoelectric layers. In addition, there are no radially applied forces. As such the neutral axis position for the receive mode $z_{m}$ reduces to the following:

$$
z_{m}=\frac{\left[\sum_{i=1}^{n} Y_{0, i}^{\prime} z_{i} h_{i}\right]}{\left[\sum_{i=1}^{n} Y_{0, i}^{\prime} h_{i}\right]}
$$

\section{RESULTS AND DISCUSSION}

In order to verify the derived theoretical model in the previous section, a unimorph PMUT structure is considered as shown in Figure 1(a). The PMUT is composed of silicon as a structural layer and PZT as a piezoelectric layer. Table 1 summarizes the material properties and geometric dimensions of the PMUT analyzed in this paper. The theoretically derived neutral axes in transmit and receive modes were calculated as $1.41 \mu \mathrm{m}$ and $2.10 \mu \mathrm{m}$, respectively. It is observed that the neutral axis in the receive mode is closer to the geometric mid-plane scaled by Young's modulus and the thickness of each layer. On the other hand, the neutral axis location in the transmit mode is deeper into the structural layer. Since the piezoelectric layer applies an additional stress to the structural layer, the location of the zerostrain plane is pushed farther into the silicon.

Table 1: Geometric and mechanical properties of the structural layer (Silicon) and piezoelectric layer (PZT), which form the PMUT.

\begin{tabular}{|l|l|c|c|c|}
\hline & \multicolumn{1}{|c|}{ Description } & Silicon & PZT & Units \\
\hline $\boldsymbol{Y}_{\boldsymbol{0}}$ & Young's Modulus & 169 & 63 & {$[\mathrm{GPa}]$} \\
\hline $\boldsymbol{v}$ & Poisson's ratio & 0.3 & 0.3 & \\
\hline $\boldsymbol{r}_{\boldsymbol{m}}$ & Radius & 100 & 100 & {$[\mathrm{\mu m}]$} \\
\hline $\boldsymbol{h}$ & Thickness & 4 & 0.5 & {$[\mathrm{\mu m}]$} \\
\hline $\boldsymbol{d}_{\boldsymbol{3 1}}$ & $\begin{array}{l}\text { Piezoelectric Charge } \\
\text { constant }\end{array}$ & - & 171 & {$[\mathrm{pm} / \mathrm{V}]$} \\
\hline \hline
\end{tabular}

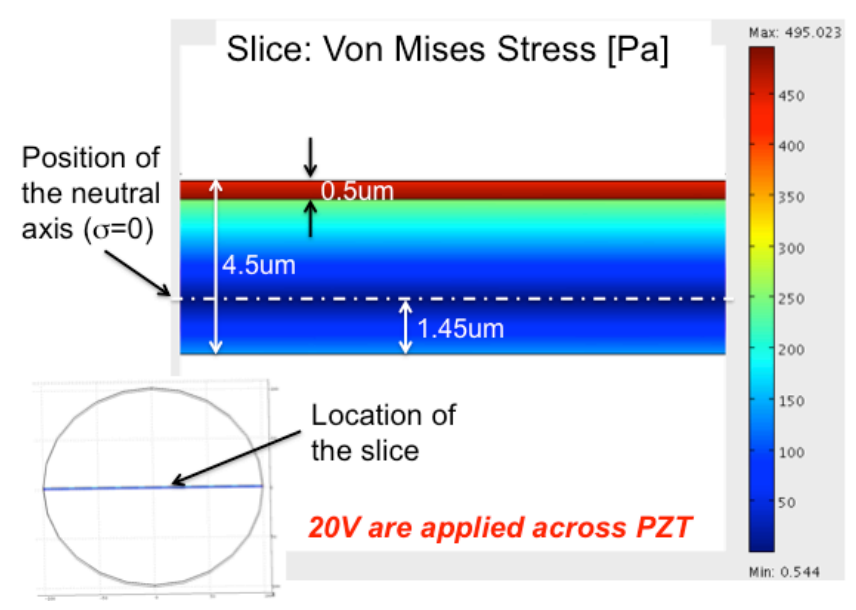

Figure 2: FEM using COMSOL of a PMUT in transmit mode. The PMUT is composed of $0.5 \mu \mathrm{m}$ thick PZT layer on top of $4 \mu \mathrm{m}$ thick Silicon layer. An electric potential of $20 \mathrm{~V}$ is applied across the piezoelectric layer. The Von Mises stresses are measured and the neutral axis is located at $1.45 \mu \mathrm{m}$ using a cross-section at the center of the membrane.

COMSOL Multiphysics was used as the Finite Element Modeling (FEM) tool for the zero-strain plane simulation [14]. The PMUT plate model was developed by extruding the mesh generated from a $2 \mathrm{D}$ circle. The resolution of the vertical mesh layers in the z-direction was set at 10 layers $/ \mu \mathrm{m}$ in order to ensure accurate extraction of the neutral axis position. The Von Mises stresses were calculated across the PMUT cross-sectional area at the center of the plate and the zero-stress location was identified. To simulate a PMUT in transmit mode, only an electric potential of 
$20 \mathrm{~V}$ was applied across the top piezoelectric layer. In the receive mode simulation, both the top and bottom electrodes sandwiching the piezoelectric layer were grounded to ensure that no electric field is developed. An impinging acoustic pressure of $100 \mathrm{kPa}$ was applied. Figures 2 and 3 show the Von Mises stresses extracted from COMSOL simulation of a PMUT plate with the same structure composition and material properties as the theoretically analyzed plate in transmit and receive configuration, respectively. The neutral axes positions were subsequently inferred from the zero-stress locations as $1.45 \mu \mathrm{m}$ and $2.16 \mu \mathrm{m}$. The values extracted from the FEM match closely with the theoretically calculated locations. An error of less than $3.6 \%$ between the theoretical and FEM approach validate the credibility of the developed model.

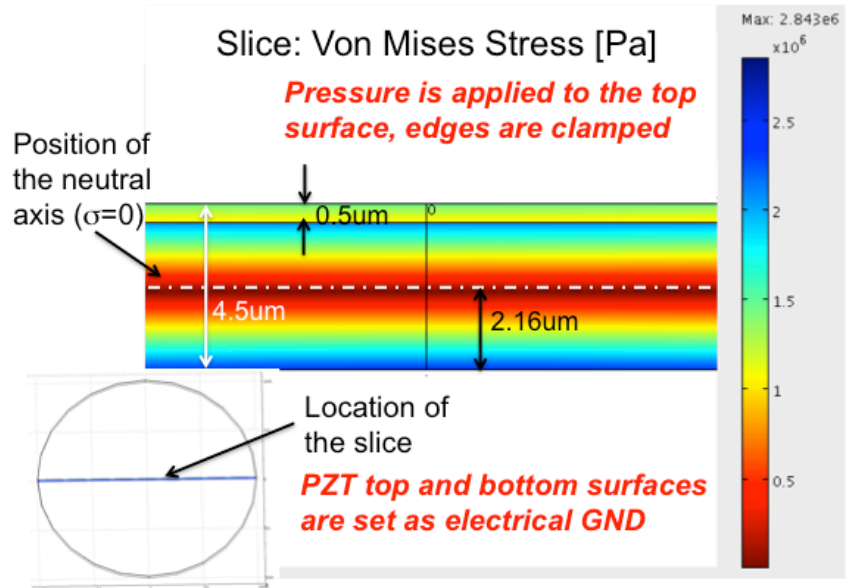

Figure 3: FEM using COMSOL of a PMUT in the receive mode. A pressure level of $100 \mathrm{kPa}$ is applied to the membrane. The Von Mises stresses are measured and the neutral axis is located at $2.16 \mu \mathrm{m}$ using a cross-section at the center of the membrane.

\section{CONCLUSION}

The neutral axis location for a circular PMUT composed of multiple structural and piezoelectric layers was investigated for the first time. The PMUT plate was modeled using the classic plate theory, where in-plane stress conditions, isotropic materials, and non-slip interfaces between the layers were assumed. The externally applied forces and moments per unit width were calculated as a function of the plate radii of curvature, layer geometric and material properties, including the thickness, Young's modulus, and Poisson's ratio, as well as the piezoelectric charge constant and the electric field across each piezoelectric layer. The general position of the neutral axis, or the position of zero bending strain, was derived for any externally applied radial moment and force per unit width, the material properties and layer dimensions, as well as the electric fields applied on each piezoelectric layer. Specific solutions of the neutral axis position were extracted for the transmit case, where only external electric fields are applied, and receive mode, where only external pressure is present. The analytical model was verified using COMSOL Multiphysics with a unimorph PMUT composed of a silicon structural layer and PZT piezoelectric layer. The location of zero Von Mises stress plane was used to locate the neutral axis from the FEM. The agreement between the theoretical and experimental tools was better than $3.6 \%$.

\section{ACKNOWLEDGEMENTS}

This project is supported by Masdar Institute of Science and Technology, Abu Dhabi, UAE grant no. 6923443 under the cooperative agreement between Masdar Institute of Science and Technology and Massachusetts Institute of Technology.

\section{REFERENCES}

[1] G. S. Kino, Acoustic Waves: Devices, Imaging, and Analog Signal Processing, Prentice Hall, 1987.

[2] A. R. Selfridge and S. Gehlbach, "KLM Transducer Model using Transfer Matrices," in Proceeding of IEEE Ultrasonics Symposium, pp. 875-877, 1985.

[3] W. A. Smith, "The Role of Piezocomposites in Ultrasonic Transducers," in Proceeding of IEEE Ultrasonics Symposium, pp. 755-766, 1989.

[4] B. T. Khuri-Yakub and O. Oralkan, "Capacitive Micromachined Ultrasonic Transducers for Medical Imagining and Therapy, "Journal of Micromechanics and Microengineering, vol. 21, no. 5, pp. 1-11, May 2011.

[5] P. C. Eccardt and K. Niederer, "Micromachined Ultrasound Transducers with Improved Coupling Factors from a CMOS Compatible Process," Ultrasonics, vol. 38, pp. 774-780, 2000.

[6] J. H. Mo, A. L. Robinson, D. W. Fitting, F. L. Terry, and P. L. Carson, "Micromachining for Improvement of Integrated Ultrasonic Transducer Sensitivity," IEEE Transactions on Electron Devices, vol. 37, no. 1, pp. 134-140, January 1990.

[7] S. Shelton, M. L. Chan, H. Park, D. Horsely, B. Boser, I. Izyumin, R. Przybyla, T. Frey, M. Judy, K. Nunan, F. Sammoura, and K. Yang, "CMOS-Compatible AlN Piezoelectric Micromachined Ultrasonic Transducers," 2009 IEEE International Ultrasonics Symposium (IUS), pp. 402405, Rome, Italy, September 20-23, 2009.

[8] P. Muralt, N. Ledermann, J. Baborowski, A. Barsegar, S. Gentil, S. Petitgrand, A. Bosseboeuf, and N. Setter, "Piezoelectric Micromachined Ultrasonic Transducers based on PZT Thin Films," IEEE Transactions on Ultrasonics, Ferroelectrics, and Frequency Control, vol. 52, no. 12, pp. 2276-2288, December 2005.

[9] J. J. Bernstein, L. Finberg, K. Houston, L. C. Niles, H. D. Chen, L. E. Cross, K. K. Li, and K. Udayakumar, "Micromachined High Frequency Ferroelectric Sonar Transducers," IEEE Transactions on Ultrasonics, Ferroelectrics, and Frequency Control, vol. 44, no. 5, September 1997.

[10] G. Perçin and B. T. Khuri-Yakub, "Piezoelectrically Actuated Flextensional Micromachined Ultrasound Transducers - I: Theory," IEEE Transactions on Ultrasonics, Ferroelectrics, and Frequency Control, vol. 49, no. 5, pp. 573-584, May 2002.

[11] D. DeVoe and A. P. Pisano, "Modeling and Optimal Design of Piezoelectric Cantilever Microactuators," Journal of Microelectrimechanical Systems, vol. 6, no. 3, pp. 266-270, September 1997.

[12] M. S. Weinberg, "Working Equations for Piezoelectric Actuators and Sensors," Journal of Microelectromechanical Systems, vol. 8, no. 4, December 1999.

[13] T. Ikeda, Fundamentals of Piezoelectricity, Oxford University Press, 1990.

[14] http://www.comsol.com

\section{CONTACT}

*Firas Sammoura, Massachusetts Institute of Technology, 77 Massachusetts Avenue, Building 41-211; Tel: +1-510-529-9142; Fax:+1-617-252-2982; sammoura@MIT.edu 